\title{
PROOF OF THE KONTSEVICH NON-COMMUTATIVE CLUSTER POSITIVITY CONJECTURE
}

\author{
DYLAN RUPEL
}

\begin{abstract}
We extend the Lee-Schiffler Dyck path model to give a proof of the Kontsevich non-commutative cluster positivity conjecture with unequal parameters.
\end{abstract}

Let $k$ be any field of characteristic zero. For any $r \in \mathbb{Z}_{>0}$, consider the following $k$-linear automorphism of the skew-field $K=k(x, y)$ of rational functions in non-commutative variables $x$ and $y$ :

$$
F_{r}:(x, y) \mapsto\left(x y x^{-1},\left(1+y^{r}\right) x^{-1}\right) .
$$

Our main result is the following.

Theorem 1 (Kontsevich conjecture). For any $r_{1}, r_{2} \in \mathbb{Z}_{>0}$ and any $k \geq 0$, the elements $x_{k}=\underbrace{F_{r_{1}} F_{r_{2}} F_{r_{1}} \cdots}_{k}(x)$ are given by non-commutative Laurent polynomials in $x$ and $y$ with non-negative integer coefficients.

Remark 2. Using a symmetry argument, Theorem 1 implies an analogous statement for $y_{k}=\underbrace{F_{r_{1}} F_{r_{2}} F_{r_{1}} \cdots}_{k}(y)$.

The Laurentness of these expressions was established by Usnich [4 for $r_{1}=r_{2}$ and by Berenstein-Retakh [1] for general $r_{1}, r_{2}$. The positivity was shown by Di Francesco-Kedem [2] for $r_{1} r_{2}=4$ and by Lee-Schiffler [3] for $r_{1}=r_{2}$. We follow the Lee-Schiffler approach in this note.

Fix integers $r_{1}, r_{2} \in \mathbb{Z}_{>0}$. Our proof will make use of two-parameter Chebyshev polynomials $U_{k, j}, k, j \in \mathbb{Z}$, defined recursively by: $U_{-1, j}=0, U_{0, j}=1, U_{k+1, j+1}=r_{j} U_{k, j}-U_{k-1, j-1}$, where $r_{j}= \begin{cases}r_{1}, & \text { if } j \text { is odd; } \\ r_{2}, & \text { if } j \text { is even. }\end{cases}$

From now on we will work under the assumption $r_{1} r_{2} \geq 5$. The cases $r_{1} r_{2} \in\{1,4\}$ were settled in [4] and [2] and the remaining cases $r_{1} r_{2} \in\{2,3\}$ are given explicitly at http://pages.uoregon.edu/drupel/dyck_examples.pdf.

Fix $n \geq 2$. Consider the rectangle $R_{n} \subset \mathbb{Z}^{2}$ with corner vertices $(0,0)$ and $\left(U_{n-3,1}-U_{n-4,2}, U_{n-4,2}\right)$. When $R_{n}$ lies in the first quadrant, a Dyck path is a lattice path in $R_{n}$ starting at $(0,0)$ and taking North or East steps to end at $\left(U_{n-3,1}-U_{n-4,2}, U_{n-4,2}\right)$ such that the path never crosses the main diagonal of $R_{n}$ and the slope of each subpath beginning at $(0,0)$ does not exceed the slope of the main diagonal. Here we consider a vertical edge to have slope $\infty$. We modify this definition slightly when $R_{n}$ lies in the second quadrant by replacing the East step with a diagonal $(-1,1)$-upstep and considering vertical edges to have slope $-\infty$. When $n=2, R_{n}$ lies in the fourth quadrant and we use a diagonal $(1,-1)$-downstep. We will call a Dyck path maximal if no subpath of another Dyck path lies closer to the main diagonal. Write $D_{n}$ for the maximal Dyck path in $R_{n}$. The next Lemma follows by induction from the definitions.

Lemma 3. Denote $\epsilon_{k}:=\max \left\{0,2-r_{k-1}\right\}, \delta_{k}:=\epsilon_{k}+2 \epsilon_{k-1}+1$ for $k \in \mathbb{Z}$. Suppose $k-\delta_{k} \geq 4$. Then the Dyck path $D_{k}$ consists of $r_{k-\epsilon_{k-1}}-\delta_{k}+1$ copies of $D_{k-1-\epsilon_{k-1}}$ followed by a copy of $D_{k-1-\epsilon_{k-1}}$ with its first $D_{k-1-\delta_{k}}$ removed.

Let $U_{n}=\max \left\{\left|U_{n-3,1}\right|,\left|U_{n-4,2}\right|\right\}$ be the number of edges in $D_{n}=\left(\omega_{0}, \alpha_{1}, \omega_{1}, \alpha_{2}, \ldots, \alpha_{U_{n}}, \omega_{U_{n}}\right)$, where the vertices of $D_{n}$ are labeled by $\omega_{0}, \omega_{1}, \ldots, \omega_{U_{n}}$ and $\alpha_{i}$ is the edge connecting $\omega_{i-1}$ and $\omega_{i}$. Let $i_{1}, \ldots, i_{U_{n-4,2}}$ denote the increasing sequence so that $\alpha_{i_{j}}$ makes an upward step. We will write $\nu_{0}, \ldots, \nu_{U_{n-4,2}}$ for the sequence of vertices satisfying $\nu_{0}=(0,0)$ and $\nu_{j}=\omega_{i_{j}}$. 
Definition 4. For $i<j$ denote by $s_{i j}$ the slope of the line from $\nu_{i}$ to $\nu_{j}$ and by $s$ the slope of the main diagonal of $R_{n}$. For $0 \leq i<k \leq U_{n-4,2}$ let $\alpha(i, k)$ be the subpath of $D_{n}$ from $\nu_{i}$ to $\nu_{k}$ labeled/colored as follows:

(1) If $s_{i t} \leq s_{n}$ for all $t$ with $i<t \leq k$, then $\alpha(i, k)$ is called a Dyck prefix (blue).

(2) If $s_{i t}>s_{n}$ for some $t$ with $i<t \leq k$, then

(a) if the smallest such $t$ is of the form $i+U_{m, 2}-w U_{m-1-\epsilon_{m-1}, 2}$ for some integers $1 \leq m \leq n-4$ and $1 \leq w<r_{m-\epsilon_{m-1}}-\delta_{m}$, then $\alpha(i, k)$ is called an $(m, w)$-Dyck suffix (green).

(b) otherwise, $\alpha(i, k)$ is called a short suffix (red).

Write $\mathcal{P}\left(D_{n}\right)=\left\{\alpha(i, k): 0 \leq i<k \leq U_{n-4,2}\right\} \cup\left\{\alpha_{1}, \ldots, \alpha_{U_{n}}\right\}$ for the set of admissible subpaths of $D_{n}$. For $\beta \subset \mathcal{P}\left(D_{n}\right)$ we define the support $\operatorname{supp}(\beta) \subset D_{n}$ in the natural way. We will use the term hook for the supports of the subpaths $\alpha(k, k+1)$. It will be convenient to refer to a hook as type 1,2 , or 3 depending on whether the horizontal displacement from the bottom to the top of the hook is $r_{2}-1, r_{2}-2$, or $r_{2}-3$, respectively.

Call $\beta \subset \mathcal{P}\left(D_{n}\right)$ an overlapping collection if there exists either $\alpha(i, k), \alpha\left(i^{\prime}, k^{\prime}\right) \in \beta$ which share a vertex or $\alpha_{j}, \alpha(i, k) \in \beta$ with $\alpha_{j} \in \alpha(i, k)$. We will need the following $K$-valued weightings on non-overlapping collections.

Definition 5. Write $\varepsilon_{i}=\left\{\begin{array}{ll}1 & \text { if } \alpha_{i} \text { is vertical; } \\ 0 & \text { otherwise. }\end{array}\right.$ For each non-overlapping collection $\beta \subset \mathcal{P}\left(D_{n}\right)$ define

$$
\beta_{[i]}= \begin{cases}y^{r_{1}-\varepsilon_{i}} x^{-1}, & \text { if } \alpha_{i} \notin \operatorname{supp}(\beta) ; \\ y^{-\varepsilon_{i}} x^{-1}, & \text { if } \alpha_{i} \in \beta \text { and } \alpha_{i} \text { is not diagonal; } \\ x^{1} y^{-1} x^{-1}, & \text { if } \alpha_{i} \in \beta \text { and } \alpha_{i} \text { is diagonal with an upstep; } \\ x^{0} y^{1}, & \text { if } \alpha_{i} \in \beta \text { and } \alpha_{i} \text { is diagonal with a downstep; } \\ x^{0} y^{0}, & \text { if } \alpha_{i} \in \alpha(j, k) \in \beta \text { is horizontal; } \\ x^{h} y^{-1} x^{-1}, & \text { if } \alpha_{i} \in \alpha(j, k) \in \beta \text { is the last edge of a hook of type } h .\end{cases}
$$

We have the following refinement of Theorem 1

Theorem 6. Suppose $r_{1}, r_{2} \in \mathbb{Z}_{>0}$. Write $q=x y x^{-1} y^{-1}$. Then for $n \geq 2$ we have $x_{n-1}=\sum_{\beta \in \mathcal{F}\left(D_{n}\right)} q \prod_{i=1}^{U_{n}} \beta_{[i]}$, where the product is taken in the natural order and the sum ranges over the set $\mathcal{F}\left(D_{n}\right)$ of non-overlapping collections $\beta \subset \mathcal{P}\left(D_{n}\right)$ subject to the conditions:

$C 1$ : if $\alpha_{i}$ is diagonal, then $\alpha_{i}$ is supported on $\beta$;

$C 2$ : if $\alpha(i, k) \in \beta$ is a short suffix, then the preceding non-diagonal edge of $\nu_{i}$ is supported on $\beta$;

$C 3:$ if $\alpha(i, k) \in \beta$ is an $(m, w)$-Dyck suffix, then at least one of the preceding $U_{m-1,1}-w U_{m-2-\epsilon_{m-1}, 1}$ non-diagonal edges of $\nu_{i}$ is supported on $\beta$.

Example 7. For $r_{1}=2, r_{2}=3, n=5$ we have $U_{2,1}=5, U_{1,2}=2$ and so $R_{5}$ and $D_{5}$ are given by: $\quad \ldots:$. We have the following expression for $x_{4}$ :

$$
\begin{aligned}
x_{4}= & q x y^{-1} x y^{-1} x^{-1}+q x y^{-1} x^{-1}\left(1+y^{2}\right) x^{-1}\left(1+y^{2}\right) y^{-1} x^{-1}+q\left(1+y^{2}\right) x^{-1}\left(1+y^{2}\right) x^{-1} y^{-1} x y^{-1} x^{-1}+ \\
& +q\left(1+y^{2}\right) x^{-1}\left(1+y^{2}\right) x^{-1}\left(1+y^{2}\right) y^{-1} x^{-1}\left(1+y^{2}\right) x^{-1}\left(1+y^{2}\right) y^{-1} x^{-1}
\end{aligned}
$$

where a factor of $1+y^{2}$ indicates an edge which may be either included in or excluded from the corresponding admissible collection of labeled/colored subpaths. We present several examples for $r_{1} r_{2}=5$, enumerating all admissible collections with their monomials, at http://pages.uoregon.edu/drupel/dyck_examples.pdf.

Proof of Theorem [6: We divide the proof into a series of lemmas. First we make the following definitions.

Definition 8. Define the set $\tilde{\mathcal{F}}\left(D_{n}\right)$ of non-overlapping collections $\beta \subset \mathcal{P}\left(D_{n}\right)$ subject to conditions $C 1$ and $C 2$. Define $\mathcal{T} \geq u\left(D_{n}\right) \subset \tilde{\mathcal{F}}\left(D_{n}\right)$ to consist of those $\beta$ satisfying the following condition only for $m \geq u$ :

$C 3^{o p}$ : there exists integers $i, k, w, m$ such that $\alpha(i, k) \in \beta$ is an $(m, w)$-Dyck suffix and none of the preceding $U_{m-1,1}-w U_{m-2-\epsilon_{m-1}, 1}$ non-diagonal edges of $\nu_{i}$ are supported on $\beta$. 
Lemma 9. If $m \geq n-3$, there do not exist $i, w\left(1 \leq w<r_{m-\epsilon_{m-1}}-\delta_{m}\right)$ so that $\min \left\{t: i<t \leq U_{n-4,2}, s_{i, t}>s\right\}$ is of the form $i+U_{m, 2}-w U_{m-1-\epsilon_{m-1}, 2}$. In particular, for any $n \geq 2$, the set $\mathcal{T} \geq n-3\left(D_{n}\right)$ is empty.

Proof. We assume $\epsilon_{m-1}=0$; the case $\epsilon_{m-1}>0$ follows from this one. Since $w<r_{m}-1-\epsilon_{m}$, we have

$$
U_{m, 2}-w U_{m-1,2} \geq U_{m, 2}-r_{m} U_{m-1,2}+\left(2+\epsilon_{m}\right) U_{m-1,2}=\left(2+\epsilon_{m}\right) U_{m-1,2}-U_{m-2,2} \geq U_{m-k, 2} \text {, for } k \geq 1 .
$$

Now if $m \geq n-3$ and $\tau:=\min \left\{t: i<t \leq U_{n-4,2}, s_{i, t}>s\right\}=i+U_{m, 2}-w U_{m-1,2}$, then $\tau \geq i+U_{n-4,2}$. But this contradicts $\nu_{U_{n-4,2}}$ being the highest labeled vertex in $D_{n}$.

Let $z_{0}=x_{0}=x$ and for $n \geq 2$ write $z_{n-1}=\sum_{\beta \in \tilde{\mathcal{F}}\left(D_{n}\right)} q \prod_{i=1}^{U_{n}} \beta_{[i]}$. For each integer $\ell$ we will use a parenthesized exponent ${ }^{(\ell)}$ to denote a quantity with each $r_{k}$ replaced by $r_{k+\ell}$.

Lemma 10. Suppose $n \geq 2$. Then $z_{n}^{(1)}=F_{r_{2}}\left(z_{n-1}\right)+\sum_{\beta \in \mathcal{T} \geq 1\left(D_{n+1}^{(1)}\right) \backslash \mathcal{T} \geq 2\left(D_{n+1}^{(1)}\right)} q \prod_{i=1}^{U_{n+1}^{(1)}} \beta_{[i]}$.

Proof. This follows from a study of how the $\left(1+y^{r_{2}}\right)^{-1}$ terms cancel in $F_{r_{2}}\left(z_{n-1}\right)$. In particular, we make the following observations. The sum of the weights of a colored hook and the corresponding full hook of uncolored edges gives rise to a Laurent monomial under $F_{r_{2}}$. An edge $\alpha$ in the support of $\beta$ gives rise to a colored hook of type 1, 2, or 3 corresponding to the edge $\alpha$ being horizontal, vertical not followed by a diagonal, or vertical followed by a diagonal, respectively. A missing edge $\alpha$ gives rise to all collections of uncolored edges in a hook of type 1, 2, or 3 corresponding to the edge $\alpha$ being horizontal, vertical not followed by a diagonal, or vertical followed by a diagonal, respectively.

Now consider an uncolored hook with a missing horizontal edge, followed by $d$ included horizontal edges, and then an included vertical edge. Under $F_{r_{2}}$ the weight of this configuration gives rise to the weights of all collections of horizontal edges in a hook of type 1 with an included vertical edge followed by $d$ colored hooks of type 1 and then a colored hook of type 2 . The sum is accounting for the included vertical edge in this case.

In the following Lemma we consider a $D_{3}$ with its first $D_{2}$ removed as a single vertical edge and for $\epsilon_{3}=1$ we consider a $D_{4}$ with its first $D_{2}$ removed as a vertical edge followed by a $(-1,1)$-diagonal edge.

\section{Lemma 11.}

(1) Suppose $k-\epsilon_{k-1} \geq 5$. Then the weight of a missing $D_{k-2}$ with its first $D_{k-3-\epsilon_{k-3}}$ removed followed by a colored $D_{k}$ simplifies to the weight of a colored $D_{k-1-\epsilon_{k-1}}$.

(2) Suppose $k-\epsilon_{k-1} \geq 5$. Then the weight of a missing $D_{k-2}$ followed by a colored $D_{k-1-\epsilon_{k-1}}$ simplifies to the weight of a missing $D_{k-2}$ with its first $D_{k-3-\epsilon_{k-3}}$ removed.

(3) Suppose $m-\delta_{m} \geq 0$. Then for $1 \leq w<r_{m-\epsilon_{m-1}}-\delta_{m}$, the weight of an $(m, w)$-Dyck suffix preceded by $U_{m-1,1}-w U_{m-2-\epsilon_{m-1}, 1}$ missing non-diagonal edges is equal to the weight of an $(m, w+1)$-Dyck suffix preceded by $U_{m-1,1}-(w+1) U_{m-2-\epsilon_{m-1}, 1}$ missing non-diagonal edges.

Proof. Parts (11) and (2) follow from a simultaneous induction using Lemma 3 in the induction step. Part (3) follows from (11), (2), and Lemma 3 .

Corollary 12. Suppose $m-\delta_{m} \geq 0$. Then for $1 \leq w<r_{m-\epsilon_{m-1}}-\delta_{m}$, the weight of an $(m, w)$-Dyck suffix preceded by $U_{m-1,1}-w U_{m-2-\epsilon_{m-1}, 1}$ missing non-diagonal edges is equal to $q^{-1}$.

Proof. We work by induction, the case $m-\delta_{m}=0$ is easy to check by hand. It follows from Lemma 3 that the hook sequences of an $\left(m, r_{m-\epsilon_{m-1}}-\delta_{m}\right)$-Dyck suffix and an $(m-1,1)$-Dyck suffix are the same. Then one easily checks that $U_{m-1,1}-\left(r_{m-\epsilon_{m-1}}-\delta_{m}\right) U_{m-2-\epsilon_{m-1}, 1}=U_{m-2,1}-U_{m-3-\epsilon_{m-2}, 1}$, the case $\epsilon_{m-1}>0$ following from the case $\epsilon_{m-1}=0$. The result now follows by induction using Lemma 1113,

We remind that a parenthesized exponent ${ }^{(\ell)}$ denotes a quantity with each $r_{k}$ replaced by $r_{k+\ell}$. In particular, note that $F_{r_{2}}\left(x_{k}\right)=x_{k+1}^{(1)}$. 
Lemma 13. Let $u \geq 1$ and $n \geq u+4$. Then

$$
F_{r_{2}}\left(\sum_{\beta \in \mathcal{T} \geq u\left(D_{n}\right) \backslash \mathcal{T} \geq u+1} q \prod_{i=1}^{U_{n}} \beta_{[i]}\right)=\sum_{\beta \in \mathcal{T} \geq u+1} \sum_{\left(D_{n+1}^{(1)}\right) \backslash \mathcal{T} \geq u+2\left(D_{n+1}^{(1)}\right)} q \prod_{i=1}^{U_{n+1}^{(1)}} \beta_{[i]} .
$$

Proof. The proof follows by simultaneous induction with Lemma 14. We will assume $n=u+4$, the case $n>u+4$ follows from this one using a similar argument. Also we restrict to the case $\epsilon_{n-1}=0$, the case $\epsilon_{n-1}>0$ follows by a similar argument.

From Lemma 3, we can see that $D_{n}$ begins with $w$ copies of $D_{n-1}, 1 \leq w<r_{n}-1-\epsilon_{n}$, and the vertex $\nu_{w U_{n-5,2}}$ is the ending vertex of the last $D_{n-1}$. Now $\alpha\left(w U_{n-5,2}, U_{n-4,2}\right)$ is the only $(n-4, w)$-Dyck suffix of $D_{n}$ and so $\beta \in \mathcal{T}^{\geq n-4}\left(D_{n}\right)$ implies $\alpha\left(w U_{n-5,2}, U_{n-4,2}\right) \in \beta$ and none of the preceding $U_{n-5,1}-w U_{n-6,1}$ non-diagonal edges are contained in $\beta$. Note that $w U_{n-4,1}-U_{n-5,1}+w U_{n-6,1}=r_{2} w U_{n-5,2}-U_{n-5,1}$ and so the lowest vertex of these missing edges is $\omega_{r_{2} w U_{n-5,2}-U_{n-5,1}}$. Then Lemma 3 implies the subpath of $D_{n}$ from $\omega_{0}$ to $\omega_{r_{2} w U_{n-5,2}-U_{n-5,1}}$ consists of $w-1$ copies of $D_{n-1}$, followed by $r_{n-1}-1$ copies of $D_{n-2}$, and then $w-1$ copies of $D_{n-3}$. We will define $j_{i}$ for $0 \leq i \leq 2 w+r_{n-1}-3$ so that the $\nu_{j_{i}}$ are the endpoints of these copies. Any subpath $\alpha(i, k)$ can be decomposed as $\alpha\left(i, j_{e}\right), \alpha\left(j_{e}, j_{e+1}\right), \ldots, \alpha\left(j_{e+\ell}, k\right)$ where all but the first are Dyck prefixes. It is easy to see that $\alpha\left(i, j_{e}\right)$ has the same label/color as $\alpha(i, k)$ and if $\alpha(i, k)$ was an $\left(m, w^{\prime}\right)$-Dyck suffix then so is $\alpha\left(i, j_{e}\right)$.

Combining the above considerations we see that $\sum_{\beta \in \mathcal{T} \geq u\left(D_{n}\right) \backslash \mathcal{T} \geq u+1} q \prod_{i=1}^{U_{n}} \beta_{[i]}$ can be rewritten as:

$$
\begin{aligned}
& \sum_{w=1}^{r_{n}-2-\epsilon_{n}} q\left(\sum_{\beta \in \mathcal{F}\left(D_{n-1}\right)} \prod_{i=1}^{U_{n-1}} \beta_{[i]}\right)^{w-1}\left(\sum_{\beta \in \mathcal{F}\left(D_{n-2}\right)} \prod_{i=1}^{U_{n-2}} \beta_{[i]}\right)^{r_{n-1}-1}\left(\sum_{\beta \in \mathcal{F}\left(D_{n-3}\right)} \prod_{i=1}^{U_{n-3}} \beta_{[i]}\right)^{w-1} q^{-1} \\
= & \sum_{w=1}^{r_{n}-2-\epsilon_{n}} q\left(q^{-1} x_{n-2}\right)^{w-1}\left(q^{-1} x_{n-3}\right)^{r_{n-1}-1}\left(q^{-1} x_{n-4}\right)^{w-1} q^{-1},
\end{aligned}
$$

where the equality follows from Lemma 14, Applying $F_{r_{2}}$ and noting that $F_{r_{2}}(q)=q$ completes the proof.

Lemma 14. Suppose $n \geq 3$. Then

$$
x_{n-1}=z_{n-1}-\sum_{m=5}^{n} \underbrace{F_{r_{1}} F_{r_{2}} F_{r_{1}} \cdots}_{n-m}\left(\sum_{\beta \in \mathcal{T} \geq 1\left(D_{m}^{(m-n)}\right) \backslash \mathcal{T} \geq 2\left(D_{m}^{(m-n)}\right)} q \prod_{i=1}^{U_{m}^{(m-n)}} \beta_{[i]}\right)=\sum_{\beta \in \mathcal{F}\left(D_{n}\right)} q \prod_{i=1}^{U_{n}} \beta_{[i]} .
$$

Proof. This follows from simultaneous induction with Lemma [13 as in the proof of [3, Lemma 20].

Acknowledgements. This project was initiated while the author was visiting the new Center for Mathematics at Notre Dame in June of 2011. We would like to thank CMND for their hospitality.

\section{REFERENCES}

[1] A. Berenstein and V. Retakh, "A Short Proof of Kontsevich Cluster Conjecture." C. R. Math. Acad. Sci. Paris 349 (2011), no. 3-4, 119122.

[2] P. Di Francesco and R. Kedem, "Discrete Non-Commutative Integrability: Proof of a Conjecture of M. Kontsevich." Int. Math. Res. Not., 2010, no. 21, 40424063. (doi:10.1093/imrn/rnq024)

[3] K. Lee and R. Schiffler, "Proof of a Positivity Conjecture of M. Kontsevich on Non-Commutative Cluster Variables." Preprint: math.QA/1109.5130, 2011.

[4] A. Usnich, "Non-Commutative Laurent Phenomenon for Two Variables." Preprint math.AG/1006.1211, 2010.

Department of Mathematics, University of Oregon, Eugene, OR 97403

E-mail address: drupel@uoregon.edu 